\title{
Mercadeo: El reto de las Revistas Científicas
}

\author{
Yasmile Navarro R.* \\ Maritza E. Vanegas C."*
}

\section{Resumen}

Se realizó un estudio exploratorio sobre tres aspectos relativos a la comercialización de revistas cientificas: el mercado y su segmentación, los responsables de marketing y la planificación del producto final con sentido de mercadeo, partiendo de las teorlas de mercadotecnia y considerando el tratamiento dado en las revistas a las que se tuvo acceso. Se hizo una revisión bibliográfica, observación directa de las revistas y entrevistas no estructuradas como instrumentos para la recolección de información. Dado que el tema ha sido trabajado por pocos autores, la base teórica fundamental parte de Jabal, Holtje y Garcla Dlaz. Se hace una recopilación que permita proseguir la investigación con el fin de contribuir con el desarrollo de la producción y divulgación cientifica en la Universidad del Zulia (LUZ).

Palabras claves: Mercadeo, Revistas Cientfficas, Universidad.

\section{Marketing: A defiance in Scientific Review}

\begin{abstract}
An explorative study was carried out three aspects of comercialization of scientific journals: the market and its segments, those responsible for marketing and planing of the final product in relation to marketing, taking into consideration marketing theories and the treatment given by each joumal reviewed. A bibliographic review and direct observations of the journals, and non-structured personal interviews were carried out to collect data.
\end{abstract}

Recibido: 31-01-96 . Aceptado: 27-03-96

- Contador Públlco, Becario Académico de la Facultad de Clenclas Económicas y Soclales de la Universidad del Zulla, cursante de la Especialización en Costos. Telt. 061-618243-596554.

** Economista, Investigadora adscrita al instituto de Investigaciones de la Facultad de Clenclas Económicas y Soclales de la Unlversidad del Zulia, tesista de la Maestría en Gerencla Pública. 
This topic has been investigated by few authors, therefore the theoretical base was taken from Jabal, Holtje and Garcla Dlaz. This paper allows further research and helps greatly to scientific knowledge.

Key words: Periodical Marketing, Scientif Review, University.

\section{Introducción}

El avance cientffico influye notablemente en el desarrollo económico, politico, cultural y social. De la producción de conocimientos depende el progreso de la sociedad, pero no es suficiente investigar y descubrir, es necesario además escribir y divulgar los resultados obtenidos.

En la Universidad del Zulia (LUZ) hasta finales de la década de 1980, habla poca divulgación de los progresos cientificos, no existian suticientes estímulos a la investigación y menos aún a la difusión. La situación era más grave en las Ciencias Sociales.

Sin embargo, las politicas del Consejo Nacional de Investigaciones Cientificas, especialmente el Programa de Promoción al Investigador (PPI), la aplicación de criterios de productividad cientifica en la asignación del presupuesto universitario, la gestión rectoral de las actuales autoridades, asi como la del Consejo de Desarrollo Científico y Humanístico (CONDES), han creado condiciones propicias para el cambio.

Los investigadores están asimilando cada vez más, aunque en algunos casos lentamente, la trascendencia del trabajo escrito como via tradicional y casi única, de comunicación efectiva con el lector mundial, y en consecuencia, la escritura como parte esencial de la profesión del cientfifico, ya que la etapa más importante de una investigación es la pre- paración de los resultados para publicación en el medio adecuado. (Crespo, 1987).

Por tratarse de un campo poco explorado en el pals el estudio se fundamenta en la revisión de los escasos materiales bibliográficos especificos disponibles, aún cuando existe abundante producción general sobre mercadeo. En consecuencia, a partir de la base térica se centró la atención en la observación de las revistas a fin de captar en el producto final los elementos claves de mercadeo. La información anterior fue complementada con entrevistas personales a miembros de Comités Editoriales de algunas revistas de la Universidad del Zulia.

Este aporte intenta contribuir con ol desarrollo cientifico-técnico del pals, al facilitar el mercadeo de revistas a fin de lograr prestigio e impacto nacional $\theta$ internacional, incentivando a nuestros investigadores y docentes a escribir sus avances cientfficos y publicar oportunamente en lugares válidos. Se pretende asi colaborar con el CONDES, que ha demostrado un gran interés por promover la investigación y las publicaciones cientifficas lo que supone determinar $y$ aplicar mecanismos y estrategias de mercadeo.

\section{Comercialización de publicaclones especializadas}

En general, la comercialización es un proceso en el cual se realizan una 
serie de actividades, que conducen los bienes y servicios desde el productor al consumidor.

Bajo el concepto gerencial moderno, el mercadeo de las revistas cientfficas no se puede reducir a garantizar la producción y entrega a los usuarios de las mismas. Debe ser el producto de un examen cuidadoso de lo que necesitan los usuarios de información cientifica, on combinación armónica con las necesidades de difusión de los adelantos de la ciencia. Son estos factores los que deben considerarse en la creación y diseño de las revistas.

Otro aspecto importante es encontrar la manera en que se puede producir la publicación con la mayor eficiencia, esto es, editar al menor costo posible la cantidad de revistas necesarias y con la calidad requerida, de acuerdo a las expectativas de venta, sin olvidar la reserva indispensable para los servicios postventa.

De igual manera es necesario buscar la forma de comercializar las publicaciones con el mayor beneficio cientifico e institucional, sin perder de vista lo económico. Esta consideración es importante por cuanto el mercadeo de revistas cientificas en Latinoamérica, a diferencia del mundo industrializado, no está aứn en condiciones de relevar el objetivo de rentabilidad. Es necesario priorizar la divulgación de los avances cientificos y tecnológicos y la visibilidad de la institución que los genera. (Maclauchlan, 1988).

La evaluación periódica de los resultados debe permitir tomar las decisio. nes pertinentes para ofrecer y distribuir las mejores publicaciones en cada área.
El mercadeo supone una serie de actividades que se inician antes de la edición: planeación de la revista (formato, nombre, empaque, etiqueta, financlamiento, costo y tiempo de edición, expectativas de los usuarios y autores, etc.), diseño de las pollticas de comercialización (competencia, canales de distribución, precios, promoción, etc) y la ejecución del mercadeo propiamente tal.

La mezcla de mercadeo adecuada es diferente en cada caso; se debe seleccionar de acuerdo a las características de la revista, de los autores que en ella publican y de los usuarios. El mejor plan se determinará estudiando diferentes alternativas.

El plan anual de mercadeo facilita que las estrategias a largo plazo puedan ejecutarse para asegurar el logro de las metas. Por lo tanto, debe tener una orientación definida y además, permitir enfrentar los problemas especificos que requieran solución inmediata; debe ser flexible, a fin de posibilitar durante su ejecución, los ajustes que la evaluación sugiera.

\section{El mercado de Revistas Clentiflcas}

En sentido general, el mercado supone un espacio real o figurado de encuentro entre la oferta y la demanda. Ahora bien, en sentido estricto, se considera que el mercado se compone de aquella demanda que determina la producción necesaria.

El mercado de revistas cientfficas está constituido por profesionales y/o instituciones con necesidades de información sobre los avances de la producción 
de conocimientos. Sin embargo, no es suficiente que exista la necesidad de conocer el desarrollo científico y revistas que lo difundan, es necesario que se combinen otros elementos de acuerdo a la naturaleza del producto especffico, para asegurar la satisfacción de los usuarios, lo cual está en relación directa con la utilidad que genera y espera el productor.

En este caso se trata de discutir la comercialización de publicaciones especializadas, esto es, aquellos documentos que son portadores de información, que satisfacen la necesidad de un individuo 0 colectividad, y que se hacen públicos a través de cualesquiera de los soportes tradicionales: papel, microficha, microfilm, cinta magnética, disco duro, disco óptico, otc. (García Diaz, 1994).

Las revistas cientfficas pueden ser de distintos tipos: primarias, de resúmenes o secundarias, de progresos cientificos o tecnológicos y de intormación. Nuestra atención apuntará a las revistas primarias por ser el medio considerado válido para la divulgación científica. Según Crespo (1987:1-4), el trabajo cientifico no se publica válidamente si se publica on mal lugar, sólo se acepta si se publica en lugar apropiado, el cual debe ser una revista primaria indizada, esto es, registrada en las bases de datos de la especialidad, y que esté disponible oportunamente para la comunidad científica.

La materia prima que conforma la médula de las revistas son los articulos o memorias cientificas originales, aunque también pueden publicarse notas técnicas, comunicaciones cortas sobre descubrimientos importantes 0 cartas al editor con opiniones relevantes de los lectores.
Los artículos científicos describen resultados originales de investigación y contribuyen a ampliar el conocimiento o la comprensión de un problema; deben redactarse de tal manera que cualquier investigador competente pueda reproducir el experimento y obtener los resultados descritos, repetir las observaciones, cálculos o deducciones teóricas del autor y juzgar sus conclusiones. (Crespo, 1987:7).

El acceso al mercado de revistas cientfficas tiene como elementos claves: la periodicidad, oportunidad, calidad editorial y científica, disponibilidad en bibliotecas o hemerotecas públicas, asequibilidad en los servicios de recuperación de información (bases de datos), etc.

En principio, el mercado de las revistas cientificas editadas en LUZ es el conjunto de investigadores, dependencias, instituciones y empresas nacionales o internacionales que tienen necesidad de información sobre los adelantos cientifico-tecnológicos que se generan en esta casa de estudios, en cada área de conocimiento en la cual las revistas se espocializan.

Sea cual fuere el producto a comercializar, es necesario conocer el mercado y los submercados a los cuales va dirigido, esto es segmentar el mercado: decidir qué parte del mismo, qué clase de consumidores finales se adecúa a las posibilidades de nuestro producto, en razón de sus caracteristicas, asi como la capacidad de producción y distribución de la organización que lo genera. (Jabal, 1982:53).

Actualmente, el segmento de mercado de las revistas cientfficas está conformado por el grupo de investigadores que ha desarrollado cuando menos un 
nivel mínimo de cultura de la divulgación cientffica. Es escasa la utilización de revistas entre estudiantes y profesores universitarios; generalmente son sugeridas por los autores a sus alumnos de postgrado y eventualmente en pregrado. Por otra parte, no está garantizada la disponibilldad de las revistas propias de cada especlalidad en las diferentes instituciones públicas o privadas correspondientes, ni siquiera en los centros de investigación y bibliotecas especializadas. Incluso, por ejemplo, la Biblioteca Central de la Universidad del Zulia no cuenta con colecciones completas de cada una de las revistas que la Universidad ha editado; peor aún, algunos comités editoriales tampoco poseen la colección completa.

Cada subdivisión del mercado es un grupo homogéneo al cual por sus características similares puede llegarse más tácilmente (Holtje, 1991:13). Para llegar a esos submercados con mayor eficiencia y productividad se deben considerar algunos elementos de mercadotecnia que podrían aplicarse al proceso de comercialización de nuestro producto.

En el caso de las revistas cientificas, esta homogeneidad es producto de la consideración de criterios de segmentación relacionados con los usuarios y/o con la revista y sus editores.

Entre los relativos al usuario, tenemos: Nivel Educativo y Especialidad Profesional; nivel económico-social (ingresos, zona geográfica); edad y sexo; hábitos de compra, lectura y consulta; cantidad y tipo de usuarios potenciales y riesgo por estacionalidad de la demanda.

Los factores relacionados con la revista a comercializary con los editores, que deben considerarse para acceder al segmento del mercado son: a) Ambito de circulación: sólo para miembros, nacional o internacional. b) Presentación: formato, diseño, color, cantidad de páginas y calidad de la impresión. c) Gama de productos y servicios post-venta. d) Competencia: posición en el mercado de otras revistas afines, diferenciación de esta revista respecto a las otras. Interesa no sólo la competencia desde el punto de vista de los usuarios, sino en cuanto al interés de los autores por publicar en ella. e) Distribución: propia, intermediarios, mixta.f) Regulaciones: Derecho de Autor, International Standard Serial Number (ISSN), Depósito legal en el Instituto Autónomo Biblioteca Nacional, cuota para otras bibliotecas públicas y especializadas (postgrado, centros de investigación, facultades y/o escuelas, organismos gremiales, etc).

Segmentar el mercado de revistas cientificas implica llegar a un público espocffico, editando una publicación más especializada; "cuando el costo de la segmentación es muy elevado, puede resultar desventajosa si no se tiene una población suficientemente amplia donde las ventas compensen los gastos" (Jabal, 1982:53).

Cada caso de segmentación debe ser analizado como tal. Veamos cómo se comportan algunos criterios en un caso especifico. Aunque en Venezuela hay cierta variedad de publicaciones cientificas en ciencias sociales, puede ocurrir que se aspira editar una revista en el campo gerencial, que hasta este momento no existia en el pais, a pesar de la indiscutible trascendencia de los temas gerenciales.Sin embargo, existen revistas comerciales promovidas por las aso- 
ciaciones de ejecutivos o por los gremios profesionales como la Revista Investigación y Gerencia, donde han publicado conjuntamente académicos y ejecutivos; a pesar de su importancia, estas revistas no reúnen los requisitos necesarios para ser consideradas revistas cientificas, ya que publican básicamente trabajos de carácter técnico $u$ opiniones fundamentadas en la experiencia, y no resultados de investigación; tampoco son arbitradas, requisito fundamental de las revistas cientificas, aún cuando tienen amplia acogida en el mercado. En el caso concreto de la Revista Investigación y Gerencia, recientemente se ha suspendido su publicación. (Gamboa et al, 1995).

La inexistencia de revistas gerenciales arbitradas evidencia que en Venezuela la cultura cientifica en dicha área está en proceso de construcción; esto implica la necesidad de conjugar esfuerzos inter-institucionales para impulsar el desarrollo de la producción y divulgación cientlfica en el campo gerencial y para promover su distribución y el uso efectivo del conocimiento difundido.

Una revista de gerencia no sólo incentiva el desarrollo científico en este campo históricamente signado por el pragmatismo, sino que permite propiciar una mayor vinculación con el nivel gerencial de empresas e instituciones gubernamentales, facilitando la contrastación teoria-práctica. Ahora bien, los cargos gerenciales son ejercidos por profesionales de diversas disciplinas y lo mismo ocurre con los postgrado en gerencia; esto implica que el segmento potencial del mercado no incluye sólo a los investigadores y docentes del área.
Por otra parte, por sus caracteristicas, el nivel ejecutivo exige una revista de alta calidad en cuanto a su presentación, asi como artículos redactados en un lenguaje directo y preciso, que formulen claramente su objeto de estudio y sus referencias conceptuales. En forma similar, tanto los ejecutivos como la academia, pueden requerir no sólo la revista impresa, sino una gama de productos y servicios post-venta, tales como: edición en microformas, diskette, CD-Rom, incorporación de tablas de contenido de la revista en la red de redes (Intemet) para facilitar el intercambio electrónico de información, suministro de separatas, información sobre eventos gerenciales, cursos de postgrado, etc. (Gamboa et al, 1995).

Con la investigación del mercado se obtiene información sobre los compradores de cada segmento y sub-segmento posible. La información debe ser accesible, mensurable y posible de evaluar; es decir, que se tenga acceso tanto al mercado como a datos sobre hábitos de compra, por ejemplo, a través de fuentes de información tales como asociaciones industriales y comerciales, informes de consumidores, etc.; que sean cuantificables las tendencias de los hábitos de compra y que puedan ser sometidas a un análisis económico en cuanto a volumen de venta proyectado y costo de ventas (Jabal, 1987:14).

\section{Responsables de marketing de publicaclones clentíficas}

En una empresa la elaboración del plan de mercadeo es responsabilidad del departamento correspondiente, el cual en 
el caso de las revistas universitarias deberla ser la Editorial (EDILUZ).

Sin embargo, por sus caracteristicas peculiares, generalmente esta labor ha sido asumida por el comité editor, formado en principio por personas con alto nivel cientifico pero que no están capacitadas para optimizar el trabajo de comercialización; ésto a pesar de tratarse de un sólo tipo de producto y editado en cantidades casi siempre limitadas, puesto que el tiraje en la mayorla de los casos conocidos no sobrepasa los mil ejemplares. Aún cuando los comités editoriales realizan la distribución de las revistas, no se han propuesto cumplir sistemáticamente con las actividades propuestas por la mercadotecnia.

La institución contaba con la editorial de la Universidad del Zulia (EDILUZ), dependencia que debiera encargarse de dichas actividades, ya que existen normas que determinan sus responsabilidades, as! como los lineamientos de promoción y distribución de publicaciones, divulgando el conocimiento a través de la edición y distribución de medios impresos que le sirven de soportea las funciones básicas de LUZ, docencia, investigación y extensión.

El boletín de normas y procedimientos establece las pautas que regirán la comercialización de libros, revistas y demás publicaciones editadas por la Universidad del Zulia y de aquellas otras en cuya edición ésta participe o cuya difusión asuma. En el mismo boletín hay toda una explicación sobre los tipos de ventas y pasos a seguir, instrumentos para el registro de información (recibos, comprobantes de venta, consignación, etc), lineamientos de promoción y distribución, sistema contable, solicitud de promoción, venta $\theta$ impresión por parte de las dependencias y procedimientos para las cobranzas respectivas. (LUZ, 1982).

EDILUZ tenla las siguientes funciones: editar las publicaciones con su respectiva inscripción, promocionarlas, distribuirlas e imprimir otros productos como atiches, tarjetas, diseño de carátulas, etc. Sin embargo, EDILUZ no cumple con todas sus funciones por muchas causas, tales como: a) Falta de una buena organización; b) Problemas gerenciales; c) Algunos equipos eran obsoletos; d) No se utilizaban los convenios con librerías y distribuidores cabalmente; $\theta$ ) Corrupción (desmantelamiento de equipos).

Estos problemas redundaron en una ineficiente actividad de EDILUZ; $\theta$ dita algunos libros con bastante retraso, por lo cual algunas veces los autores se ven en la necesidad de pedir que se les termine la obra en talleres privados. La comercialización de sus propias ediciones es deficiente, pues se observan centenares de libros viejos acumulados en los almacenes del taller que no salieron a la venta por falta de promoción y distribución; por lo tanto era aún más dificil que promocionara y distribuyera las publicaciones de otras dependencias.

En consecuencia, la Universidad del Zulia decidió en 1995 reestructurar la Editorial, eliminando sus funciones de producción, para convertirla en un Consejo de Publicaciones con características propias y con suficiente jerarqula academica y organizativa, que someta a arbitraje los trabajos cuya publicación sea solicitada, con el fin de enviarlos a un proceso de impresión en talleres privados, cues- 
tión que aún no se está aplicando. En este momento EDILUZ está haciendo un esfuerzo para optimizar su funcionamiento y tiene previsto abrir su propia libreria.

El Consejo de Publicaciones tiene como función: 1) Evaluar y actualizar poIíticas editoriales válidas para toda la universidad. 2) Asesorar y coordinar la correcta aplicación de las políticas y planes que el mismo apruebe. 3) Proponer el anteproyecto de presupuesto para todas las publicaciones de LUZ. 4) Autorizar cada publicación y los nuevos proyectos de revistas especializadas.

Las fuentes de ingreso del Consejo de Publicaciones serían el producto de la venta de las publicaciones, asignación del Consejo Universitario, ingresos propios y presupuestarios de las Autoridades Rectorales, recursos del CONDES, de las Facultades, contribución de instituciones extrauniversitarias, donaciones y otras.

La nueva polltica editorial tiene las características de: Integridad, incentivos y prioridad a las publicaciones perí́dicas dentro de un plan de democratización de la divulgación cientifica.

De manera que, todavia no existe una unidad institucional que se pueda responsabilizar de la comercialización de las revistas cientificas. Por lo tanto volvemos a la situación inicialmente planteada: El comité editor debe continuar la tarea de comercializar su revista, por lo cual debe prepararse para ello.

\section{Planiflcación de la revista desde el punto de vista de marketIng}

La primera consideración necesaria respecto al mercadeo de las revistas cientificas es tomar en cuenta que sus posibilidades y sus problemas se inician desde el momento que se concibe la idea de publicar una revista. Puesto que no se trata simplemente de colocar en el mercado el producto elaborado, sino de producir un medio de divulgación cientffica que responda a las necesidades de autores y lectores potenciales. Por lo tanto la planificación del producto final debe hacerse con una concepción clara de mercadeo.

El diseño del producto final implica definir los atributos físicos de la revista: formato, portada, cantidad de páginas, calidad del papel, secciones que contendrá además de los artículos, (reseñas de libros, información sobre eventos), diagramación, envases y embalaje, aunque no se limita a ellos. La elección acertada de estos elementos, para evitar ajustes posteriores que afectan la homogeneidad relativa de la revista, puede tener gran importancia en la futura distribución.

Entre los atributos físicos uno de los más importantes es el formato; la exploración indica que la mayoría de las revistas en Ciencias Sociales utilizan un formato de 1/8 mientras las de Ciencias Naturales prefieren el de 1/4. El formato está directamente relacionado con la ubicación de las revistas en los anaqueles de las bibliotecas; de manera que un cambio de formato afecta la conservación de la serie en un mismo lugar o la apreciación estética del conjunto, y puede dar lugar a que se cancele la suscripción. (Garcla Dlaz, 1994).

La elección del papel también afecta la distribución. Ciertos medios profesionales están habituados a revistas de alta calidad en su apariencia y pueden subes- 
timar una divulgación de gran calidad cientifica, pero presentada pobremente. Por otra parte, el papel tiene un peso determinado, el cual incide en los costos de distribución postal. En consecuencia, se requiere sopesar ambas variables.

Envase es el recipiente que se encuentra en contacto directo con el producto para proteger sus características físicas y quimicas. Es un elemento importante en la mercadotecnia para presentar una imagen agradable y atractiva al consumidor $\theta$ inspirarle confianza on la calldad del producto. (Garcia Dlaz, 1994). De manera que el envasado debe asegurar su conservación, pero también debe hacer una contribución promocional. En el caso de las revistas pueden considerarse dos tipos de envases: los sobres de envio y la cobertura de protección, que puede ser de papel o de plástico.

Las decisiones concernientes al nombre del producto, las marcas patentadas, los nombres de marcas, las garantlas de diversos tipos y la vida de mercado antlcipada del producto, forman parte también del mismo y deben desarrollarse tomando en cuenta la forma en que éstas se relacionan con la satisfacción de las necesidades del consumidor. (Holtje, 1991:2).

Las revistas clentfficas son un producto muy peculiar que no puede limitarse a considerar las necesidades de los lectores potenciales, sino también las de los autores que aportan la materia prima editorial. El Comité Editor como productor de las revistas, cumple una función de intermediación entre autores y lectores: presta a unos y otros el serviclo de difusión de los avances científlcos. En conse. cuencia, el nombre de la revista debe constituir un vehículo para la identificación del tipo de conocimiento que ella difunde; en otras palabras, es la primera via para atraer a quienes suministran los insumos a la revista -los autores-y para captar lectores.

Lo anterior significa que la selección del nombre es fundamentalmente un problema de mercadeo; la designación de medios de divulgación con nombres ambiguos o muy generales, puede atraer a muchos pero dificulta el acercamiento de los especialistas en el área. Sin embargo, una revista que se inicia, a menos que cuente con un amplio inventario de autores de cierto nivel y preferiblemente con cierto "colchón editorial" (reserva de articulos para publicar), no puede optar por un nombre demasiado especifico. La especialización y más aún la subespeciallzación, generalmente es una etapa posterior.

Ahora bien, desde el punto de vista de mercadeo llamamos marca a cualquier clase de nombre o símbolo destinado a identiflcar un producto, con la intención de diferenciarlo de otros. (Jabal, 1982:430). Es evidente que el nombre de la revista es la marca primordial de este producto.

A su vez, la márca debe registrarse, esto es protegerse legalmente contra la copla o el fraude. La protección legal consiste en facultar exclusivamente al propietario para utilizarla. (Jabal, 1982:43). La marca de la revista está registrada, cuando su nombre aparece inscrito en el International Standard Serlal Number -ISSN-, lo cual implica la asignación de un número; además, da derecho de propledad a 
los autores de los articulos y contribuye a evitar los plagios. EI registro ISSN se realiza a través del Instituto Autónomo Biblioteca Nacional.

Por otra parte, toda revista primaria tiene que contar con una organización o institución que la patrocine (asociación académica o científica) en cuyo nombre se publica la revista y es el techo bajo el cual se desarrollan mayormente las actividades científicas de la publicación periódica. (Crespo, 1987:6) para una revista cientifica la marca es también la Institución que la respalda -tanto el centro de investigación como la Universidad- cuyo prestigio avala la rigurosidad de la publicación o la pone en duda.

En cuanto a la garantía del producto, ella está directamente vinculada a su calidad. Las empresas intentan por distintos medios aumentar y mantener la calidad de sus productos para garantizar la permanencia en el mercado; el hecho de ofrecer garantía contribuye a detectar posibles fallas de cualquier naturaleza y asi mejorar cada vez más sus productos. La calidad de las revistas, como medio de divulgación de conocimientos es de dos tipos: fisica y cientffica.

La calidad cientffica de la revista la garantiza en primer lugar el proceso de arbitraje al cual se someten los artículos publicados, cuestión que constituye una de las responsabilidades fundamentales del Comité Editorial. Por ello, como un factor indispensable de toda revista cientffica, es necesario contar con un grupo de revisores o árbitros de oficio y consultantes, constituido por el mayor número posible de investigadores especializados en los diversos campos cubiertos por el perfil de la revista, cuya evaluación garantice la calidad de los artículos recibidos para su publicación. (Crespo, 1987:6).

Si la calidad cientifica depende de los árbitros, es evidente que a la inversa, el nivel y la rigurosidad de los árbitros puede juzgarse por la calidad de los articulos que una revista pública. De manera que la garantía de la calidad científica radica en el arbitraje, cuya evidencia se expresa indicando en cada articulo la fecha en la cual fue entregado por los autores a los editores y la fecha en que fue aprobado para la publicación.

Un aspecto adicional de la calidad cientifica es la inclusión de la misma en las bases de datos de la especialidad, lo cual también debe ser garantizado por lo editores. Es preciso conocer las instituciones que procesan dichos índices de autores y abstracts y enviarles sistemáticamente cada ejemplar editado. La indización garantiza el acceso de los investigadores a la intormación sobre los articulos publicados en la revista; a través de las palabras claves, los estudiosos pueden consultar rápidamente los resúmenes de los articulos correspondientes. Lo más conveniente es utilizar las palabras claves ya incluidas en el Tesaurus.

En segundo lugar, la calidad de una revista debe juzgarse desde el punto de vista físico y de la edición: diseño integral, diagramación, formato, clase de papel, presentación tipográfica para contratapa y solapas, selección de colores y tipos de letras para texto y títulos, corrección de la impresión y encuadernación, etc. La garantla sobre estos dos últimos aspectos 
debe incluirse expresamente en la revista, en términos de sustitución de los ejemplares defectuosos.

El etiquetado es otro aspecto importante del mercadeo que se considera al planear la producción de un bien; las revistas deben tener un listado de los organismos que preparan bases de datos, suscriptores $a$ instituciones con las cuales realizan canje, a quienes debe enviarse inmediatamente cada número editado. En esos casos debe existir un sistema automatizado para preparar las etiquetas pre-impresas con el logo de la revista, el nombre y dirección de cada usuario para pegarlas en los sobres de envlo. Esto debe hacerse antes de la recepción de los ejemplares de la imprenta, a fin de agilizar la distribución. (Jabal, 1982:43).

Por otra parte, todo producto sea cual fuese su naturaleza tiene un ciclo de vida en el mercado que pasa por cuatro etapas: Introducción, crecimiento, madurez y declinación. No todos los productos pasan por todas la etapas ni éstas tienen la misma extensión. Algunos productos declinan durante el mismo proceso de introducción; otros logran mantenerse por cierto tiempo, pero simplemente sobreviven sin alcanzar la madurez; en el mejor de los casos pueden lograr su etapa cumbre, pero siempre existe el riesgo de declinación.

Desde el punto de vista de la planeación es importante reconocer las etapas por las cuales atravesará el producto y formular planes acordes a cada una de ellas. (Holtje 1991:14,37).

Por ejemplo, la etapa introductoria requiere una fuerte actividad promocio- nal, que de a conocer la revista entre el máximo posible de autores y lectores potenciales. Esta etapa se inicia antes de editarse el primer número, con el proceso de captación de artículos y con su arbitraje; se fortalece con la presentación oficial o "bautizo" de la revista, y se consolida a través de las relaciones públicas realizadas por el Comité Editor y con la utilización de diferentes medios publicitarios; también actúan como medios de divulgación de la revista las bases de datos $\theta$ indices electrónicos o impresos y los gopher universitarios de la red de redes (Internet). La duración de la etapa introductoria depende de la fuerza con que se difunda la revista y por supuesto, de su calidad.

\section{Concluslones}

El mercadeo constituye una actividad económica fundamental que adquiere caracterlsticas peculiares de acuerdo a la naturaleza del producto, en este caso, las revistas cientificas, donde se establece una relación tridimensional entre autores, editores y lectores, que es fundamental para el diseño de los planes de comercialización.

La Universidad del Zulia ha acentuado las pollticas de promoción a la investigación y a la publicación de sus resultados en revistas nacionales e intemacionales. Sin embargo, la divulgación propiamente dicha, requiere mucho más que la edición de las revistas: es indispensable fortalecer los planes de marketing que aseguren realmente la visibilidad de la Universidad del Zulia y de sus investigadores en el mundo. 
En este caso iniciamos la exploración acerca del mercado propio de las revistas y su segmentación, los responsables de la comercialización y la planificación del producto con sentido de marketing. Sin embargo, es preciso atender también aspectos como los canales de distribución y las pollticas de precios, e igualmente los costos en que se incurre al desarrollar una politica agresiva de penetración del mercado de revistas cientlficas y su correlación con los beneficlos clentfficos, institucionales y económicos que contribuyan a producir.

Debe hacerse notar que la mayorla de los responsables de la edición y comercialización de las revistas cientfficas son profesionales pertenecientes a campos ajenos a lo gerencial, lo cual incide en el desarrollo de la labor de mercadeo, por lo tanto es necesario el apoyo de los especialistas en esta disciplina a fin de fortalecer dicho proceso.

\section{Referenclas Blbllográflcas}

Crespo Garcla, Femando (1987). El Articulo Clontiflco: ¿Rutina o Mótodor. Edlclones Oensa. La Habana Cuba.
Gamboa, Teresa; Ochoa, Haydee; Rodriguez, Ellzabeth (1995). Proyecto para la Creación de la Revista Venezolana de Gorencla. LUZ. Maracalbo.

Garcla Dlaz, Ignacio (1994). Markoting do Publlcaciones Especlallzadas Impresas y Eloctrónicas. (MIMEO). INDICT. PROINF, La Habana, Cuba.

García Dlaz, Ignaclo (1995). Introducción acerca de la Problemática Nacional de la Polftica Editorial do las Publlcaclones Boriadas no Dlarlas INDICT-ACC. (MIMEO).

Holtje, Herbert (1991). Mercadotecnla. Shaum's Outline of Marketing. Tr: Hortensia Corona de Contin. Mo Graw Hill Interamericana. México.

Jabal, Juan (1982). Mercadotscnila. Altamira. S.A.I.G. España.

MeLauchlan de A., Patricia (1988). Indleadores Comparatlvos do los Resultados de la Investigación Clentífica y Tecnologlca en Amórlca Latina. Documento de Trabajo de GRADE. Número ll.

Ollver de G., Flor. La Formación de Colecclonos ds Publlcaciones Porlodlcas en Epoce do Crlels. (MIMEO).

Unlvereidad del Zulla (1882). Boletín de Normas y Procodimlentos pars la Ed|clón, Promoción y Distribuclón de las Publlcaclones. 